\title{
Pseudo-elliptic bandstop filter with 1:2:3 harmonic ratio stubs
}

\author{
J.A.G. Malherbe \\ By employing open circuited stubs with a length ratio of 1:2:3 separated by three unit elements, it is \\ shown that a pseudo-elliptic frequency response can be obtained with a compact structure. It has an \\ electrical performance comparable to that of a Cauer filter, but is realizable in etched form for very wide \\ bandwidths and very high rates of cutoff.
}

Introduction: It was previously shown that an elliptic-function-like bandstop response can be obtained by making use of open circuit shunt stubs and a series shorted stub [1], or open circuit shunt stubs and unit elements [2] with a stub length ratio of 1:2:4. Such filters can be made to have high rates of cutoff, and are realizable for bandwidth ratios of the order of 7:1. These filters have seven transmission zeros periodically interspersed, as shown in table 1.

Table 1: Positions of zeros of 1:2:4 filter

\begin{tabular}{|c|c|c|c|c|}
\hline Stub length & \multicolumn{4}{|c|}{ Position of zeros } \\
\hline$l_{0}$ & \multicolumn{4}{|c|}{$f_{0}=4 f_{1}$} \\
\hline $2 l_{0}$ & \multicolumn{2}{|c|}{$2 f_{1}$} & \multicolumn{2}{c|}{$6 f_{1}$} \\
\hline $4 l_{0}$ & $f_{1}$ & $3 f_{1}$ & $5 f_{1}$ & $7 f_{1}$ \\
\hline
\end{tabular}

The structure suffers from certain generic problems. Firstly, because there is only one transmission zero at $f_{0}$, the peaks around the centre frequency will be high. Secondly, the zeros at $f_{1}$ and $2 f_{1}$ lie too far apart, causing the first peak after cutoff to be high; this also holds for the peak between $6 f_{1}$ and $7 f_{1}$. Both these effects can be ameliorated to a certain extent, but only at the expense of the passband insertion loss, and the stopband attenuation level.

By comparison it should be pointed out that Cauer filters realized through redundant design from lumped element prototypes become non-realizable through the need for negative inductors.

A 1:2:3-Ratio Filter: For the 1:2:3 ratio filter, stub lengths are $l_{0}, 2 l_{0}, 3 l_{0}$, with resonances as shown in Table 2. There are now two transmission zeros at the centre frequency, which depress the centre maxima substantially. While there are now only five zeros, the first and second zeros lie $0.667 f_{1}$ apart, and the distance between the second zero and centre frequency is $2 f_{1}$. These spacings are very much closer to those of a filter with an elliptic function response.

Table 2: Positions of zeros of 1:2:3 filter

\begin{tabular}{|c|c|c|c|}
\hline Stub length & \multicolumn{3}{|c|}{ Position of zeros } \\
\hline$l_{0}$ & \multicolumn{3}{|c|}{$f_{0}=4 f_{1}$} \\
\hline $2 l_{0}$ & $2 f_{1}$ & & $6 f_{1}$ \\
\hline $3 l_{0}$ & $1.333 f_{1}$ & $4 f_{1}$ & $6.667 f_{1}$ \\
\hline
\end{tabular}

Filter structure The structure of the filter is shown in Fig. 1. As the lengths of the stubs are determined by the centre frequency of the filter, the remaining variables are the three stub impedances and three unit element impedances, and these can be readily adjusted to give a desired response. Due to the fact that the element lengths are all multiples of a quarter wavelength at the centre frequency, the filter responses are periodic, as for all commensurate line filters. 
The element values shown in Table 3 were obtained through optimization in MATLAB for a return loss of $S_{11}=-16 \mathrm{~dB}$ and stopband loss of $S_{21}=-35 \mathrm{~dB}$; the response of $S_{11}$ and $S_{21} v s$ frequency is shown in Fig. 2.

Table 3: Element values for 1:2:3 filter

\begin{tabular}{|c|c|c|c|c|c|c|}
\hline Line & $Z_{1}$ & $Z_{2}$ & $Z_{3}$ & $Z u_{1}$ & $Z u_{2}$ & $Z u_{3}$ \\
\hline$Z_{0}(\Omega)$ & 22.2 & 21.6 & 66.8 & 38.1 & 170 & 160 \\
\hline $\begin{array}{c}\text { Width } \\
(\mathrm{mm})\end{array}$ & 14.0 & 14.5 & 3.0 & 7.0 & 0.30 & 0.37 \\
\hline
\end{tabular}

Also shown in the same figure, are the corresponding responses for a Cauer filter C0520 [3] with $\theta=51^{\circ}, S_{11}=-16.1 \mathrm{~dB}$ and $S_{21}=-36.1 \mathrm{~dB}$, this being the prototype closest to the design prototype available. The $-3 \mathrm{~dB}$ points of the two filters are identical at $0.26 f_{0}$ and $1.74 f_{0}$ respectively; this corresponds to a relative $-3 \mathrm{~dB}$ bandwidth of 6.7 , or $148 \%$. The Cauer filter has a marginally wider $-35 \mathrm{~dB}$ stopband.

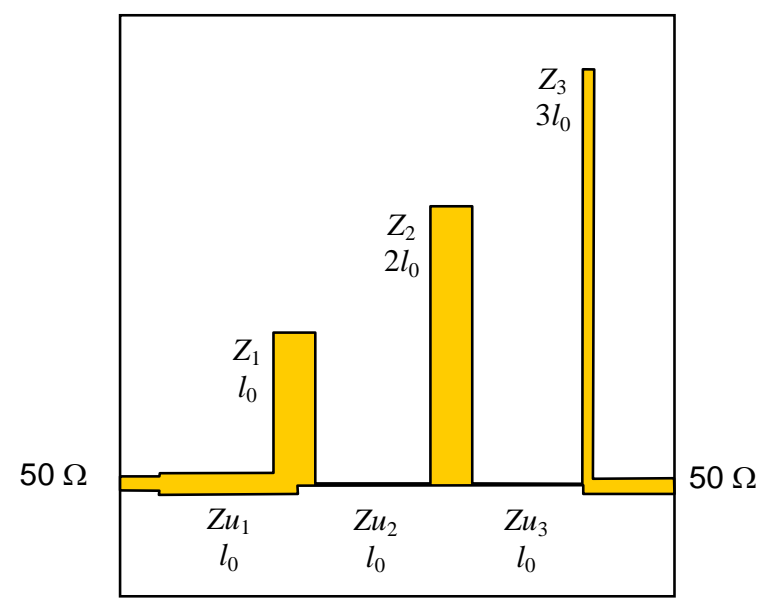

Fig. 1. Microstrip layout of 1:2:3 filter.

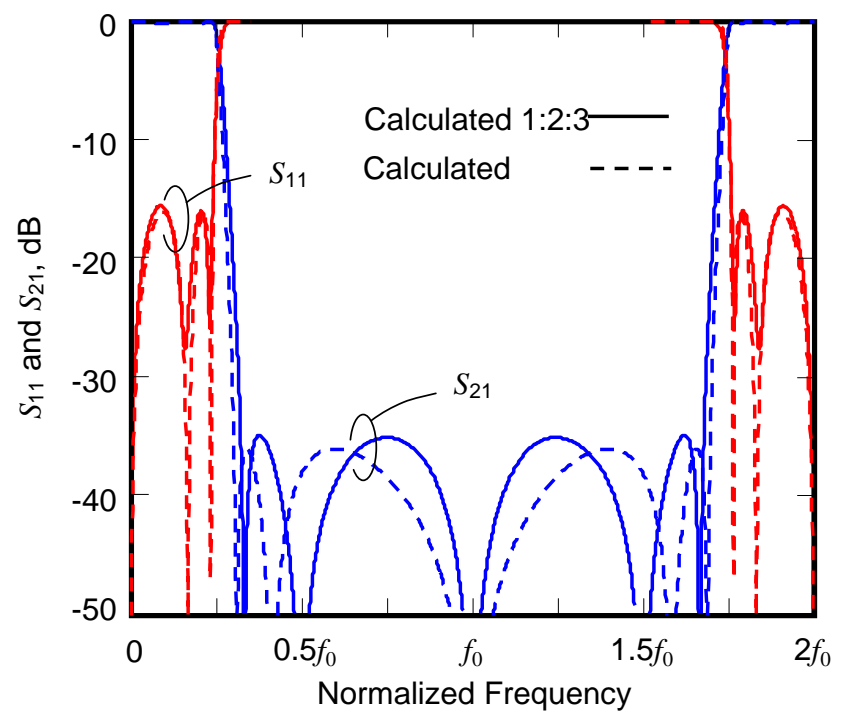

Fig. 2. Calculated response of $S_{11}$ and $S_{21}$ for the 1:2:3 filter, compared to a comparable Cauer filter.

Construction and Measurements: The filter was constructed on RT Duroid 5880 with $\varepsilon_{\mathrm{r}}=$ 2.2, and thickness $1.57 \mathrm{~mm}$. The harmonic stub length was chosen as $l_{0}=\lambda_{0} / 4=50 \mathrm{~mm}$, 
which corresponds to a centre frequency of $f_{0}=1.1 \mathrm{GHz}$. The limiting factors in the manufacture of the filter were the $170 \Omega$ and $160 \Omega$ unit elements of width $0.3 \mathrm{~mm}$ and 0.37 mm, respectively.

The filter was then modelled in Sonnet ${ }^{\circledR}$ [4]; the Sonnet model is shown in Fig. 3.

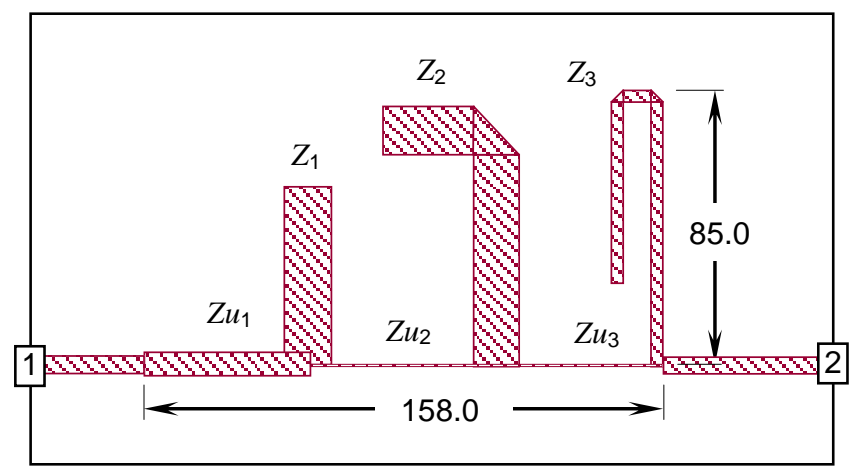

Fig. 3. Sonnet model of filter. Lines 2 and 3 are folded to conserve space.

The structure is not sensitive to line widths, but the various line lengths were adjusted to compensate for end and junction effects. The predicted response is shown in Fig. 4, and compared to the design response.

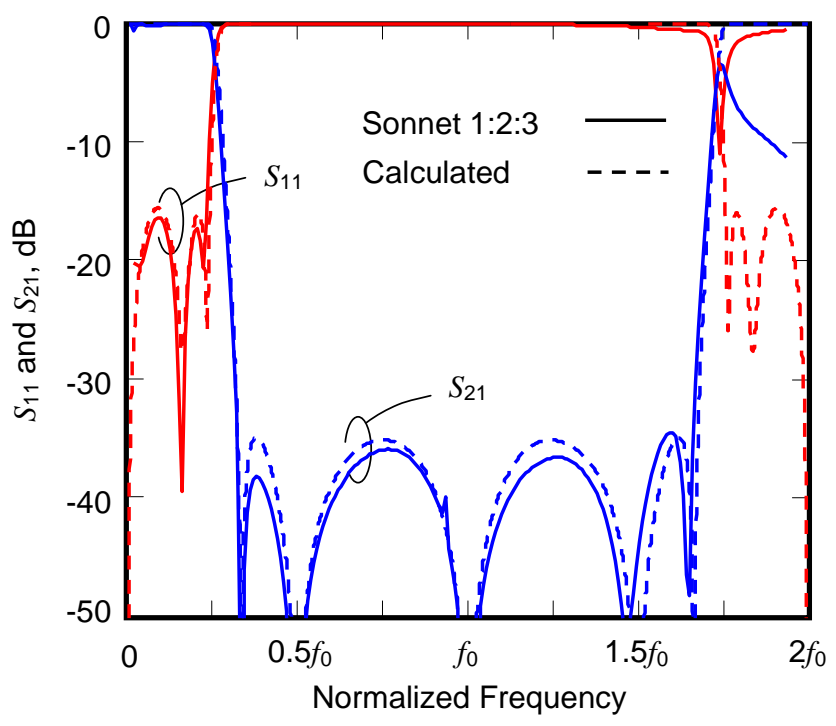

Fig. 4. Response of $S_{11}$ and $S_{21}$ for the 1:2:3 filter modelled in Sonnet, compared to a calculated prediction.

A prototype was constructed using the Sonnet dimensions, and measured on a network analyzer; the measured responses of $S_{11}$ and $S_{21}$ are shown in Fig. 5.

Conclusion: The response of the constructed filter agreed extremely well with the calculated values. At the high frequency end, beyond $2 \mathrm{GHz}$, dispersion on the microstrip lines causes the response to deteriorate, so that this type of filter is well suited to application as a pseudolowpass filter. 


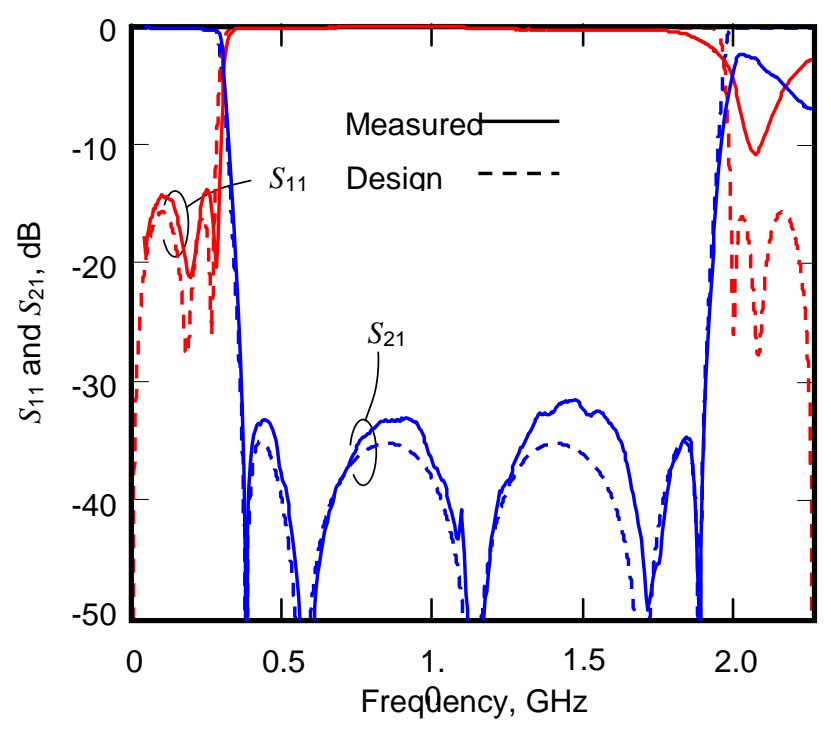

Fig. 5. Response of $S_{11}$ and $S_{21}$ for the measured prototype compared to the design response.

The structure is readily realizable, all impedances falling within the ranges that can be comfortably etched. In contrast, the Cauer filter C0520 used as comparison, necessitates an inductor of $-25 \Omega$ when designed with redundant unit elements, rendering the filter nonrealizable; this is typical of $5^{\text {th }}$ order Cauer filters of extreme bandwidths designed using the Kuroda-Levy transforms [5].

Acknowledgement: The assistance of Lukas Naudé in performing the measurements is appreciated.

J.A.G. Malherbe (Department of Electrical, Electronic and Computer Engineering, University of Pretoria, Pretoria 0002, South Africa)

E-mail: jagm@up.ac.za.

\section{References}

1 Malherbe, J.A.G.: 'Wideband Bandstop filter with Sub-Harmonic Stubs', Electron. Lett., 2011, 47, (10), pp. $604-605$.

2 Malherbe, J.A.G.: 'Wide band bandstop filter with harmonic stubs', XIII Internat. Symp. Microwave Opt. Technol. (ISMOT-2011), Prague, Czech Republic, June 20 - 23, 2011.

3 Saal, R.: 'Handbook of filter design' (AEG-Telefunken, Berlin, Germany, 1979).

4 Sonnet ${ }^{\circledR}$, High Frequency Electromagnetic Software. http://www.sonnetsoftware.com/.

5 Malherbe, J.A.G.: 'Microwave Transmission Line Filters', (Artech House, Dedham, Mass., 1979). 